\title{
Effect of tin fluoride content on the structure and properties of phosphate glass - polyamide 11 hybrids
}

\author{
Luciana Serio, David T Gawne and Yuqing Bao \\ London South Bank University, 103 Borough Road, London SE1 0AA, UK. \\ david.gawne@1sbu.ac.uk
}

\begin{abstract}
This research investigates tin-fluoride phosphate glasses as liquid-phase fillers in polyamide 11. Optimizing the tin-fluoride content was shown to reduce the glass-transition temperature of the glass down to $106^{\circ} \mathrm{C}$ and enable both constituents of the composite to be fluid during processing. During extrusion, the phosphate glass was elongated into threads and ultimately broken up into droplets. The interaction parameter between the phosphate and polyamide was found to be -0.012 due to molecular interactions and enhanced solubility of the ultrafine glass particles. All hybrids showed limited ductility due to the inability of the pinned polymer chains to be drawn into an expanding stable neck. However, there were major increases in both the Young's modulus and flexural modulus owing to the strengthening of molecular bonds by the phosphate-polyamide interactions.
\end{abstract}

Key Words: Glasses; Polymer-matrix composites; Mechanical properties; Microstructure. 


\section{INTRODUCTION}

Polymers are versatile industrial materials but their application in engineering is often restricted by property limitations such as low elastic modulus, creep and degradation. However, their properties can be tailored to some extent to satisfy a wide range of engineering applications by controlling their structure and composition. Reinforcing polymers with inorganic fillers, such as glass, is a well-established practice widely used in this regard and has a large economic advantage over synthesizing new polymeric materials. Nevertheless, conventional fillers are limited to about 40 volume $\%$ in most polymers because of the resulting high viscosity and poor processability in extrusion and injection moulding as well as limitations in extrudate quality [1,2]. A potential way to overcome these drawbacks is by developing new inorganic-organic hybrid systems characterized by the reinforcing phase being in the liquid state during polymer meltprocessing.

One class of materials that has shown promise in this context is low-temperature processable glasses. The low glass transition temperatures $T_{g}$ and softening point $T_{s}$ of these materials is achieved by using $\mathrm{P}_{2} \mathrm{O}_{5}$ instead of $\mathrm{SiO}_{2}$ as the primary glass former [49]. An important factor in the formation of low-temperature glasses is the number of crosslinkages per network-forming cation: small numbers of bridging oxygens in the structure act to decrease both $\mathrm{T}_{\mathrm{g}}$ and $\mathrm{T}_{\mathrm{s}}$. Phosphate glasses are built up from three bridging oxygens per phosphorus cation as compared with four bridging oxygens in the much higher melting silica glasses. In addition, alkali and alkaline earth oxides can be used to aid the control of thermal properties and chemical durability [4-7]. 
Research reported by a number of workers [10-16] offers the possibility that phosphate glasses can have low enough $\mathrm{T}_{\mathrm{g}}$ and $\mathrm{T}_{\mathrm{s}}$ values to be fluid alongside some thermoplastic matrices during melt processing. This would enable the prospect of mixing the components on a molecular scale and forming homogeneous hybrids: radically different structures and properties from the conventional heterogeneous glass-reinforced polymer composites.

The miscibility of polymers and glasses generally needs a negative change in the Gibbs free energy of mixing during the mixing process, which can arise from a positive entropy change and /or a negative enthalpy change. In reality, phosphate glasses and particularly polymers, are composed of large network structures with low mobilities, which inhibits increasing the entropy of mixing. In view of this, the molecular mixing of polymers and glasses needs to rely more on reductions in the enthalpy of mixing by attractive interactions between the networks, chains and side groups of the two components.

It is known that phosphate glasses can be surface nitrided $[6,7]$ and also that amine groups can bond to the surface of phosphate glasses [11-16], which are phenomena that could also lead to a reduction in the enthalpy of mixing and improve the tendency for miscibility. Work has been carried out that explores this possibility: polyamide 6 and a phosphate glass were found [11-13] to show a depression of the melting point and partial miscibility in a laboratory internal mixer. However, the work involved only one specific composition of phosphate glass and more research is required to investigate the effect of composition. This paper is directed at investigating tin fluoride glasses as liquid-phase fillers in polyamide 11 with special reference to controlling the glass-transition temperature, studying the miscibility between the phases and understanding the evolution of thermal and mechanical properties. 


\section{EXPERIMENTAL PROCEDURE}

\subsection{Materials}

Polyamide 11 (RILSAN PA11), provided by Arkema France S.A., was used as a thermoplastic matrix for the phosphate glasses. The glasses were ground to powder and sieved at $<100 \mu \mathrm{m}$, blended mechanically with powdered polyamide using a roller and compounded with a Micro-DSM extruder with an average screw speed of 100 revolutions per minute. The extruder temperature profile was set at $210 / 250 / 250{ }^{\circ} \mathrm{C}$ the total residence time during which the blend was in the chamber was set as 300s. The heating ramp to the pre-set temperatures applied was $30^{\circ} \mathrm{C} / \mathrm{min}$. The volume of the sample was kept constant at $10 \mathrm{~cm}^{3}$.

Four glasses were prepared in small $(25 \mathrm{~g})$ batches of the $\mathrm{NH}_{4} \mathrm{H}_{2} \mathrm{PO}_{4}$ reagent-grade oxide powder, $\mathrm{SnO}$ and $\mathrm{SnF}_{2}$ (Sigma-Aldrich) with molar compositional ratios $\mathrm{P}_{2} \mathrm{O}_{5} / \mathrm{Sn} 0$ of 1.5 and $\mathrm{SnF}_{2}$ contents in the range $30-60 \mathrm{~mol} \%$. The components were mixed, ground and melted for $1-2$ hours at $550^{\circ} \mathrm{C}$. Casting was undertaken by pouring the molten glass onto a metal plate for samples to be subjected to microstructural-mechanical characterization or into a graphite crucible for chemo-thermal characterization. The different casting substrates were used for ease of fabricating the test samples and any differences in cooling rate and residual stress generation were not significant owing to the subsequent annealing treatment. The latter annealing of the test samples was carried out at $120-200{ }^{\circ} \mathrm{C}$ for 2 hours and followed by storing in a desiccator. The glasses were designated as Glass A, Glass B, Glass C and Glass D. Thermal analysis of phosphate glasses was undertaken by differential scanning calorimetry (DSC) using a Mettler DSC 25 and a Perkin-Elmer DSC7 equipment: the samples were heated from 25 to $600{ }^{\circ} \mathrm{C}$ at $20^{\circ} \mathrm{C} \min ^{-1}$ in a nitrogen 
atmosphere before cooling at $30{ }^{\circ} \mathrm{C} \mathrm{min}^{-1}$ with air as the purge gas. The Littleton Softening point (the temperature at which the glass deforms visibly under its own weight) was used to evaluate softening point Ts (BS 1344-18:1981). Cylindrical glass samples (8 $\mathrm{mm}$ diameter and $13 \mathrm{~mm}$ height) were produced by pressing the dry glass powder in a cylinder mould by a hydraulic jack under a $5 \mathrm{~N} / \mathrm{mm}^{2}$ pressure. Samples of each composition were heated in a furnace from room temperature to $600^{\circ} \mathrm{C}$. The Littleton softening point of each glass was judged when the top surface started to melt. The procedure was repeated four times for each composition.

. The compositions and measured properties are given in Table 1.

\subsection{Experimental techniques}

Dogbone injected samples produced for the rheology, Dynamic Mechanical Analysis(DMA) and mechanical testing were $1 \mathrm{~mm}$ thick and 5mm wide (ISO 527) and dried at $80^{\circ} \mathrm{C}$ for 24 hours in a vacuum oven prior to testing A DMA 242 Netzsch dynamic mechanical analyser was used to determine the Tgs of the materials. The DMA test was conducted on selected injection-moulded dogbone samples at a frequency rate of $1 \mathrm{~Hz}$ under $1 \%$ strain and a ramp temperature of $2^{\circ} \mathrm{C} / \mathrm{min}$ over temperatures from $-50^{\circ} \mathrm{C}$ to $200^{\circ} \mathrm{C}$. The glass transition was determined as the maximum drop in the storage modulus when viewed on a log scale against a linear temperature scale. A concurrent peak in the $\tan \delta$ was identified and the dissipation factor was determined.

The shear viscosity of both PA and PA/Glass hybrids were determined from shear rheology using a rotational rheometer PHYSICA MCR 301. The measurements were 
performed on injection-moulded dogbone samples at $250{ }^{\circ} \mathrm{C}$ (the temperature of extrusion of the samples) and over an angular frequency range of $0.1-1000 \mathrm{rad} / \mathrm{s}$.

Thermal stability of the materials was studied by thermo-gravimetric analysis (TGA) using a Perkin Elmer TGA 7 with weight changes measured as a function of temperature using scan rates of $10^{\circ} \mathrm{C} / \mathrm{min}$. The effect of hybrid composition on weight loss at the set temperature range was monitored by a TG209 F1 Netzsch TGA. The glass and hybrid samples were heated using a furnace with an air atmosphere while the loss or gain of sample weight was monitored by a sensitive balance. Experiments were carried out within the range of the TGA $\left(100-800{ }^{\circ} \mathrm{C}\right)$ at scan rates of $10^{\circ} \mathrm{C} / \mathrm{min}$.

Water stability was assessed using the dissolution-rate method. The samples were polished prior to immersion in distilled water at $30^{\circ} \mathrm{C}$. The samples were then taken out every 2 days, dried at $100^{\circ} \mathrm{C}$ and the mass loss recorded over a total time of 14 days.

Mechanical testing involved uniaxial tensile, three point bending tests and an Olsen universal mechanical testing machine equipped with a $5 \mathrm{kN}$ load cell. The flexural bars were supported over a span of $50 \mathrm{~mm}$ with a cross-head displacement rate of $10 \mathrm{~mm} / \mathrm{min}$. Microstructure of the materials was investigated by scanning electron microscopy (SEM: HITACHI S-4300, USA). Samples were immersed in liquid nitrogen for 30 minutes, fractured and gold-coated using a BIO RAD Polaron Division SEM coating system. The glass transition temperatures of the hybrids were determined with a DMA 242 Netzsch dynamic mechanical analyser at a frequency rate of $1 \mathrm{~Hz}$ under $1 \%$ strain and a ramp rate of $2^{\circ} \mathrm{C} / \mathrm{min}$ over temperatures from $-50^{\circ} \mathrm{C}$ to $200^{\circ} \mathrm{C}$. The glass transition was determined as the maximum fall in the storage modulus on a log scale against a linear temperature scale. A peak in the $\tan \delta$ was identified and the dissipation factor measured. The melting point of the hybrids was measured using DSC by heating the samples from $25^{\circ} \mathrm{C}$ to $250^{\circ} \mathrm{C}$, 
a temperature above the melting point $\left(\mathrm{Tm}_{1}\right)$, at a rate of $20^{\circ} \mathrm{C} \min ^{-1}$. The samples were held at $250^{\circ} \mathrm{C}$ for $3 \mathrm{~min}$, then rapidly cooled at $40^{\circ} \mathrm{C} \mathrm{min}^{-1}$ to the desired crystallization temperature (Tc) and maintained at this temperature for $2 \mathrm{~min}$. Samples were heated to $200^{\circ} \mathrm{C}$ at $20^{\circ} \mathrm{C} \min ^{-1}$ for the measurement of melting point $\left(\mathrm{Tm}_{2}\right)$.

\section{RESULTS AND DISCUSSION}

\subsection{Glass Transition and softening temperature of experimental glasses}

Phosphate glasses are polymeric in nature. The tetrahedral phosphate anion $\mathrm{PO}_{4}{ }^{3-}$, as given schematically in Fig. 1(a), contains one oxygen-forming a double bond with the pentavalent phosphorus ion, which leaves the other three oxygens free to form bridges with other phosphate ions. This enables the formation of polyphosphate anions linked by oxygen bridges in linear or branched polymeric structures, as shown in Fig. 1(a). The glasses under study contain tin fluoride and tin oxide additions, which modify the structure [4-7] to give a repeat unit as in Fig.1(b). The repeat unit of polyamide 11 is given in Fig.1(c), where nitrogen as well as carbon is covalently bonded in the polymer backbone.

The introduction of fluorine into the structure results in the replacement of some of the bridging oxygens to form a $\mathrm{P}-\mathrm{F}$ terminal group, $\mathrm{PO}_{3} \mathrm{~F}$. This reduction in bridging oxygens will decrease the extent of polymerization, the network connectivity and the effective chain length. As a result, the glass structure will be expected to become more mobile as well as the glass transition temperature $T_{g}$ and the softening point $T_{s}$ reducing as the tin fluoride content increases. The experimental results in Table 1 show that $\mathrm{T}_{\mathrm{g}}$ decreases from 163 to $106{ }^{\circ} \mathrm{C}$ and $\mathrm{T}_{\mathrm{s}}$ decreases from 350 to $289{ }^{\circ} \mathrm{C}$ with increasing $\mathrm{SnF}_{2}$ content.

\subsection{Equilibrium load during extrusion process}


Monitoring the force required to extrude the hybrid materials as a function of time during the melt compounding process provides a relative measure of the resistance of the material to flow during mixing for a constant volume of compounded material and processing speed. Fig. 2 gives the loads during the extrusion process for neat PA 11 and the four hybrid materials with the same glass content of 20 vol\%. After the initial fluctuations, the curves stabilize to a plateau at an equilibrium level $\mathrm{F}_{\mathrm{e}}$. Three force-time runs were carried out for each composition for which the average equilibrium force (measured at a residence time of $120 \mathrm{~s}$ ) and standard deviation are recorded in Table 2. A representative curve is shown in Figure 2.

Table 2 gives the equilibrium extrusion forces from Fig. 2 and shows they vary markedly with composition: $1930 \mathrm{~N}$ for pure PA 11, $1880 \mathrm{~N}$ for Glass A/PA11 hybrid, $1760 \mathrm{~N}$ for GlassB/PA11 hybrid, $610 \mathrm{~N}$ for GlassC/PA11 hybrid and $350 \mathrm{~N}$ for GlassD/PA11. Table 2 also gives the effect of glass content (Glass D) on the extrusion force. This again shows a remarkable reduction of extrusion force as a result of the addition of phosphate glass to polyamide: it falls progressively from $1910 \mathrm{~N}$ for $0 \%$ glass to $350 \mathrm{~N}$ for $20 \mathrm{vol} \%$ glass. This behaviour is related to the presence of the glass in the softened or liquid state in the polyamide matrix. The Glass D/PA11 hybrid gives the lowest extrusion force indicating it has the lowest viscosity at the operating extruder temperature. This is related to the low $\mathrm{T}_{\mathrm{g}}$ and low $\mathrm{T}_{\mathrm{s}}$ of Glass D due to its high $\mathrm{SnF}_{2}$ content (60 mol\%) as shown in Table 1.

\subsection{Microstructure of hybrids}

Fig. 3 shows SEM cross-section micrographs of hybrids containing 20 vol\% of each of the four glasses in polyamide and blended under the same temperature, time and shear rate. The variations in morphology were thus expected to be due to glass composition. 
Substantial areas of the specimens were observed in the scanning electron microscope and representative images were selected for each sample. All micrographs reveal a two-phase heterogeneous hybrid of phosphate glass (light) in the polyamide matrix (dark). In the GlassA/PA11 hybrid, the glass phase (taken as Glass A in Table 1) has Tg and Ts values of 163 and $350^{\circ} \mathrm{C}$ respectively, which are too high, relative to the extruder temperature of $250^{\circ} \mathrm{C}$, for substantial melting to take place. Electron microscopy (Fig. 3a) revealed mainly angular particles with rough surfaces, which indicated that little melt-blending had taken place. Broadly similar behaviour was observed for the GlassB/PA11 hybrid with Tg and Ts values of the glass phase (Glass B) of 158 and $330^{\circ} \mathrm{C}$ respectively (Table 1 ). However, slightly more melting occurred as shown by the more rounded particles (Fig. $3 b)$.

The glass phase in the GlassC/PA11 hybrid has a $\mathrm{Tg}$ of $125^{\circ} \mathrm{C}$ and $\mathrm{Ts}$ of $310^{\circ} \mathrm{C}$ (Table 1) were lower and showed more evidence (Fig. 3c) of melting: rounded particles with smooth surfaces. The hybrid GlassD/PA11, however, exhibited a strikingly different morphology from the other hybrids: fine, rounded particles $(0.5-5 \mu \mathrm{m})$ were aligned in parallel stringers and, in view of the pronounced strain field within materials during extrusion [17], it is postulated that the observed stringers were aligned along the extrusion direction. This behaviour is related to the particularly low $\mathrm{Tg}$ and Ts values of the glass phase (Glass D in Table 1) in the hybrid: 106 and $289^{\circ} \mathrm{C}$ respectively. These relatively low critical temperatures are much closer to the temperature of the extruder $\left(250^{\circ} \mathrm{C}\right)$ and this, in combination with the high shear rate during extrusion, is expected to enable substantial flow and to elongate the glass particles into threads along the extrusion direction. The threads eventually break up into the observed multiple droplets due to Rayleigh instability involving capillary waves at the surface of the thread $[18,19]$. 
The FTIR spectra of PA11, GlassD and GlassD/PA11 hybrid are shown in Fig. 4. The GlassD/PA11 hybrid shows new peaks, not visible in the PA11 spectrum, appearing at a frequency of about $630 \mathrm{~cm}^{-1}$ (circled in Fig. 4) and these were assigned to CF deformation or $\mathrm{CF}_{3}$ asymmetric deformation [20,21]. In current research, the resonance bands are attributable to $\mathrm{SnF}_{2}$ and the interaction between PA11 and phosphate glass.

\subsection{Glass transition behaviour of PA11 and hybrids}

The glass-transition temperature of the PA11 used was determined experimentally to be $63.5^{\circ} \mathrm{C}$ and those of the phosphate glasses were measured to be in the range $105-165^{\circ} \mathrm{C}$ depending upon structure and composition. To provide additional information on $\mathrm{T}_{\mathrm{g}}$ behaviour, DMA was performed on the samples (as the loss factor tan $\delta$ provides data on the dissipation of energy). The dissipation factor as a function of temperature for PA11 and the Glass D/PA11 hybrid is given in Fig. 5 and the glass transition temperatures determined from the loss factor data of all the materials are summarized in Table 3 . The introduction of 20 vol\% to each of the four glasses into the PA matrix produced four hybrids and a decrease in $T_{g}$. Specifically, the reduction in $T_{g}$ took place with increasing $\mathrm{SnF}_{2}$ content: from $63^{\circ} \mathrm{C}$ for neat $\mathrm{PA} 11$ to $59^{\circ} \mathrm{C}$ for Glass A/PA11 and progressively down to $38^{\circ} \mathrm{C}$ for Glass D/PA11. The fact that the Tg is not in between the those of neat PA11 and the pure glass suggests that the phosphate glass-polyamide hybrid must have a significantly different structure. The $\mathrm{SnF}_{2}$ will promote the formation of the terminal group $\mathrm{PO}_{3} \mathrm{~F}$ and reduce the chain length of the phosphate glass. It is hypothesized that the smaller molecular size of the phosphate glass will allow it to occupy the intermolecular spaces between the polyamide chains and reduce the secondary-bond forces between them. This will decrease the energy needed for molecular motion and reduce the formation of hydrogen bonds between the polyamide chains. As a result, an increase in the free 
The FTIR spectra of PA11, GlassD and GlassD/PA11 hybrid are shown in Fig. 4. The GlassD/PA11 hybrid shows new peaks, not visible in the PA11 spectrum, appearing at a frequency of about $630 \mathrm{~cm}^{-1}$ (circled in Fig. 4) and these were assigned to CF deformation or $\mathrm{CF}_{3}$ asymmetric deformation [20,21]. In current research, the resonance bands are attributable to $\mathrm{SnF}_{2}$ and the interaction between PA11 and phosphate glass.

\subsection{Glass transition behaviour of PA11 and hybrids}

The glass-transition temperature of the PA11 used was determined experimentally to be $63.5^{\circ} \mathrm{C}$ and those of the phosphate glasses were measured to be in the range $105-165^{\circ} \mathrm{C}$ depending upon structure and composition. To provide additional information on $\mathrm{T}_{\mathrm{g}}$ behaviour, DMA was performed on the samples (as the loss factor tan $\delta$ provides data on the dissipation of energy). The dissipation factor as a function of temperature for PA11 and the Glass D/PA11 hybrid is given in Fig. 5 and the glass transition temperatures determined from the loss factor data of all the materials are summarized in Table 3 . The introduction of 20 vol\% to each of the four glasses into the PA matrix produced four hybrids and a decrease in $T_{g}$. Specifically, the reduction in $T_{g}$ took place with increasing $\mathrm{SnF}_{2}$ content: from $63^{\circ} \mathrm{C}$ for neat $\mathrm{PA} 11$ to $59^{\circ} \mathrm{C}$ for Glass A/PA11 and progressively down to $38^{\circ} \mathrm{C}$ for Glass D/PA11. The fact that the Tg is not in between the those of neat PA11 and the pure glass suggests that the phosphate glass-polyamide hybrid must have a significantly different structure. The $\mathrm{SnF}_{2}$ will promote the formation of the terminal group $\mathrm{PO}_{3} \mathrm{~F}$ and reduce the chain length of the phosphate glass. It is hypothesized that the smaller molecular size of the phosphate glass will allow it to occupy the intermolecular spaces between the polyamide chains and reduce the secondary-bond forces between them. This will decrease the energy needed for molecular motion and reduce the formation of hydrogen bonds between the polyamide chains. As a result, an increase in the free 
volume and molecular mobility is expected with a decrease in glass transition temperature as observed in Table 3.

No evidence of a substantial second $\mathrm{T}_{\mathrm{g}}$ peak for the glass was found in Fig. 5. The presence of only one significant peak for the hybrids was unexpected in view of the SEM observations of two phases. However, a similar result of only one $\mathrm{T}_{\mathrm{g}}$ peak was found by Urman and Otaigbe [12] for polyamide 6 and phosphate glass, although no definitive explanation was provided.

\subsection{Shear viscosity and stability}

The frequency dependence of the complex viscosity for PA11 and hybrids containing 20 vol\% of glass is shown in Fig. 6. The pure PA11 and hybrids exhibited shear thinning and a decrease of shear viscosity with higher angular frequency, which was attributed to the disruption of polymer and glass networks.

Chemical reactions due to the elevated temperatures during extrusion may also affect viscosity through their influence on molecular structure. For example, the degradation of polyamides [22] involves breakage at the $\mathrm{C}-\mathrm{N}$ bond, which creates smaller molecules and a reduction in viscosity. Thermo-gravimetric analysis (TGA) was undertaken to investigate this possibility. However, Figure 7 and Table 4 indicate that thermal stability increases rather than decreases with the addition of phosphate to polyamide and also with the amount of SnF2 in the glass. The results indicate therefore that the decrease in viscosity due to adding phosphate glass to polyamide is not related to thermal degradation phenomena. In fact, the results show that the addition of phosphate glass has the beneficial effect of improving thermal stability. For example, Figure 7 and Table 4 show that the decomposition temperatures of the GlassD/PA11 hybrid is significantly higher than those of PA1 $1\left(11^{\circ} \mathrm{C}\right.$ higher at $5 \%$ mass loss, $15^{\circ} \mathrm{C}$ at $10 \%$ mass loss and $23^{\circ} \mathrm{C}$ at $50 \%$ mass 
loss). A possible reason for the increased stability of the hybrids is the positive interaction between the phosphate and amide resulting in a more stable peptide group [22].

The instability of $\mathrm{P}_{2} \mathrm{O}_{5}$ in the presence of water may affect the behaviour of the hybrids and so tests were carried out. The weight losses of PA 11 and hybrids due to immersion in water are showed in Table 5. PA11 gave the highest dissolution rate $\left(0.0061 \mathrm{mg} \mathrm{cm}^{-2} \mathrm{~h}^{-1}\right)$, which was attributed to the hydrophilic nature of the amide groups. The presence of phosphate glasses improved the stability of the hybrids in water with the GlassD/PA11 showing the lowest dissolution rate $\left(0.0037 \mathrm{mg} \mathrm{cm}^{-2} \mathrm{~h}^{-1}\right)$. These results indicate that the viscosity reduction in the hybrids was not due to dissolution within the hybrid network.

\subsection{Miscibility of hybrids}

The depression of the melting point is a valuable indicator of the miscibility of two polymers. The thermodynamic basis for this is the exothermic heat of mixing (since the entropic contribution is small) and for miscibility, this is related to favourable interactions between the polymers. Measurements of the drop in melting point can be used to calculate a parameter $\chi$ that quantifies the extent of the interaction between the two components. This may be carried out by using the Nishi-Wang equation [23]:

$$
\left(\frac{1}{T_{b m}^{0}}-\frac{1}{T_{m}^{0}}\right)=\frac{R v_{c}}{\Delta H_{f}^{0} v_{a}} \chi_{a c} \phi_{a}^{2}
$$

where $\mathrm{R}$ is the universal gas constant, $T_{b m}^{0}$ and $T_{m}^{0}$ are the equilibrium melting points of the blend (or mixture) and the crystalline component of the blend respectively and the expression $\left(\frac{1}{T_{b m}^{0}}-\frac{1}{T_{m}^{0}}\right)$ is taken as the melting point depression. The equilibrium melting point $\mathrm{T}_{\mathrm{m}}{ }^{0}$, defined as the melting temperature of an ideal crystal having infinite crystal size. $v_{a}$ and $v_{c}$ are the molar volumes of the repeat unit of the amorphous component 
(phosphate glass in this analysis) and the crystalline component (polyamide 11 in this analysis) respectively; $\Phi a$ is the volume fraction of the amorphous component and $\Delta H_{f}^{0}$ is the heat of fusion of the crystalline component. $\chi_{a c}$ is the interaction parameter between the phosphate glass and polyamide 11 .

The Hoffman-Weeks method [11,12,23-25] was used to determine the equilibrium melting points of the pure polyamide and the hybrids. This consisted of plotting the observed melting point $\left(\mathrm{T}_{\mathrm{m}}\right)$ against the crystallization temperature $\left(\mathrm{T}_{\mathrm{c}}\right)$ for pure polyamide and the four types of phosphate glass/PA11 hybrids (GlassA/PA11, GlassB/PA11, GlassC/PA11 and GlassD/PA11). Linear plots were obtained as expected from the underlying theory and the equilibrium melting point of the material $\mathrm{T}_{\mathrm{m}}{ }^{0}$ was identified by the intersection of the plot for a given type of hybrid with the line $T_{c}=T_{m}$. The results are given in Table 6 . The equilibrium melting point $\mathrm{T}_{\mathrm{m}}{ }^{0}$ of pure PA11 was found to be $200.5^{\circ} \mathrm{C}$. The addition of 20 vol\% of each of the four glasses (A-D) to the polyamide resulted in a decrease in $\mathrm{T}_{\mathrm{m}}{ }^{0}$ of the resulting hybrid compared to PA11.

Further studies were carried out by focusing on the hybrids of Glass D (which exhibited the greatest effect) with PA11. Hybrids with different amounts of GlassD (0, 2.5, 5.0, 10.0 and $20 \mathrm{vol} \%$ ) in PA11 were prepared and the equilibrium melting points $\mathrm{T}_{\mathrm{m} 0}$ calculated with Hoffman-Weeks method are shown in Fig. 8 and Table 6. The Flory-Huggins approach (26) was then used to calculate the interaction parameter $\chi_{a c}$ between GlassD and PA11. In this analysis, the repeat unit of the phosphate glass was that proposed by Tick [5,6] (Fig. 1b) and used to calculate the molar volume $\left(\mathrm{V}_{\mathrm{a}}\right)$ of the phosphate glass. The heat of fusion of the PA11 was found experimentally to be $230 \mathrm{~kJ} / \mathrm{mol} . \mathrm{Tm}_{0}$ decreased with increasing glass content in the hybrids, from $200^{\circ} \mathrm{C}$ at $0 \%$ to $186^{\circ} \mathrm{C}$ at $20 \mathrm{vol} \%$, supporting the possibility of a thermodynamically favourable interaction between GlassD 
and PA11. The expression on the left of Equation 1 was plotted against the square of the glass volume fraction $\phi_{\mathrm{a}}^{2}$ as shown in Fig. 9 and measurement of the slope of the resulting line yielded an interaction parameter $\chi_{a c}$ of -0.012 . This indicates that there is a degree of miscibility between GlassD and PA11, which is in agreement with the decrease of both the viscosity (Fig. 6) and glass transition temperature $\mathrm{T}_{\mathrm{g}}$ (Table 3) in the GlassD/PA11 hybrid family. This result shows that a degree of interaction between the phases has occurred and partial miscibility can take place. As the melt temperature increases during blending, the increased motion of the polymer and glass segments can enable intermolecular interactions to take place. For instance, these may involve Lewis acid-base reactions involving hydrogen bonds, which could stimulate attractive interactions between the polymer and glass chains. In this context, Brow et al [8] have shown that ammonia will adsorb on the surface of phosphate glasses, implying that a similar underlying mechanism could take place in polyamide-phosphate glass systems in the liquid state.

Urman and Otaigbe [11,12] investigated polyamide 6 (PA6) and a phosphate glass (of similar composition to Glass C) and obtained an interaction parameter $\chi_{a c}$ of -0.067 . This confirms the current results that there is a positive interaction or attraction between polyamide and phosphate glass. The larger interaction parameter is attributed to the higher density (almost double) of nitrogen atoms in the polymer chain of PA6 compared with PA11 and the expected higher number of linkages between phosphate glasses and PA6 and Glass C compared with Glass C/PA11 hybrid.

A further factor affecting miscibility besides the molecular interaction between polyamide and phosphate glass, is the particle size of the glass. Small particles with high curvature exhibit an increased specific surface energy, tendency to dissolve and enhanced solubility in the surrounding matrix. This is quantified by the Gibbs-Thompson equation, which 
expresses the melting point depression $\Delta \mathrm{T}_{\mathrm{m}}$ of an isolated spherical solid particle of diameter $\mathrm{x}$ in its own liquid [27] as:

$\Delta \mathrm{T}_{\mathrm{m}}=\mathrm{k}_{\mathrm{GT}} / \mathrm{x}$

where $\mathrm{k}_{\mathrm{GT}}$ is the Gibbs-Thomson coefficient, which is dependent on interfacial geometries and materials system. The size of the phosphate glass particles in the GlassD/PA11 hybrid are expected to be sufficiently small to invoke this effect and contribute to the miscibility of this hybrid.

The results show that the addition of each of the four glasses to the polyamide decreased the $\mathrm{Tm}^{0}$ of the resulting hybrid with the lowest melting temperature being obtained for the hybrid containing 20 vol\% of Glass D. This depression of the melting point is related to the presence of $\mathrm{SnF}_{2}$ and consequent reduction in bridging oxygens, which results in a decrease in network connectivity and a more mobile structure. Moreover, the ultrafine particle size and surface energy of the glass also contribute to the miscibility and reduced melting point of the hybrids.

\subsection{Mechanical Properties}

The mechanical property parameters are given in Table 7 . The outstanding consequence of the addition of the phosphate glass to the polyamide is the reduction in elongation-atbreak, which falls from $310 \%$ for neat PA11 to $18 \%$ for GlassD/PA11. Semicrystalline thermoplastics in uniaxial tension initially show linear elastic deformation until a neck forms at the yield point and grows unstably: deformation is inhomogeneous in that it is concentrated within the neck under a falling load. The neck then stabilizes and increases in length (cold drawing) by extracting, uncoiling and straightening polymer chains from the disordered molecular chains of the amorphous regions. This mechanism continues until 
the entire sample is drawn and further stretching under a high strain-hardening rate takes place before fracture. The process involves substantial molecular chain realignment and enables extensive ductility to develop as shown in Table 7.

All of the hybrids only exhibited the initial stages of this deformation sequence: linear elasticity, yield point and unstable necking. No stable necking and cold drawing behaviour was observed. As a consequence, the hybrids showed very limited ductility as given in Table 7. On a molecular scale, this is attributed to a significant interaction between the glass and the polyamide in which glass particles pin and immobilize the chains. This inhibits the chains being uncoiled and extracted from the amorphous regions to be drawn into the expanding stable neck. The origin of this effect is the same as that quantified for the miscibility in the previous section of this paper: a significant interaction parameter and attraction between the polyamide and phosphate glass. The particularly low ductilities of Glass A/PA11 and Glass B/PA11 are exacerbated by the presence of large unmelted particles and their associated stress concentrations.

Table 7 also shows that the addition of phosphate glass to polyamide gave an increase in Young's Modulus and the Flexural Modulus, particularly for the most hybridized GlassD/PA11. This is attributed to the strengthening of the phosphate glass - polyamide bonds. The increases in yield, tensile and flexural strength shown in Table 7 are expected to relate to the significant interaction parameter and attraction between the polyamide chains and phosphate glass molecules, which restrict the uncoiling of the amorphous polyamide regions.

\section{CONCLUSIONS}


Raising the tin fluoride content of phosphate glasses substantially reduced their $\mathrm{Tg}$ and $\mathrm{Ts}$ temperatures due to the formation of $\mathrm{PO}_{3} \mathrm{~F}$ terminal groups. Processing the phosphate glasses with polyamide 11 in a micro-extruder produced predominantly heterogeneous two-phase hybrids but with a degree of miscibility owing to phosphate-polyamide molecular interactions together with the ultrafine glass particle size. All hybrids showed reduced ductility relative to neat polyamide 11 due to the absence of stable necking and cold drawing. Both the Young's modulus and flexural modulus, however, showed substantial increases owing to the strengthening of molecular bonds by the phosphatepolyamide interactions.

\section{ACKNOWLEDGEMENT}

This research received funding from the European Union Seventh Framework Programme (FP7-MC-ITN) and the authors would like to thank the Directorate-General for Science, Research and Development of the European Commission for financial support.

\section{REFERENCES}

1. J. Singh, M.Kumar, S. Kumar, S. K. Mohapatra, Properties of Glass-Fiber Hybrid Composites: A Review. Polymer-Plastics Technology and Engineering 56 (2017) 455469

2. M.M. Irina, A.I. Azmi, C.L. Tan, C.C. Lee, A.N.M Khalil, Evaluation of Mechanical Properties of Hybrid Fiber Reinforced Polymer Composites and their Architecture, Procedia Manufacturing 2 (2015) $236-240$

3. Young RT, Baird DG. The influence of processing variables on injection moulded in situ composites based on polyphenylene sulfide and a melt processable glass. Composites Part B 2000; 31: 209-221. 
4. Sanford LM, Tick PA. Tin-phosphorous oxyfluoride glasses. 1982, US Patent: $4,314,031$

5. Tick PA. Tin-phosphorous oxychloride glass containing aromatic organic compounds. 1983, US Patent: 4,379,070.

6. Tick PA. Water Durable Glasses with Ultra Low Melting Temperature. Physics and Chemistry of Glasses 1984; 25: 149-154.

7. Adalja SB, Otaigbe JU. Melt rheology of tin phosphate glasses. Applied Rheology 2001; 11: 10-8.

8. Brow RK, Zhu Y,.Day DSE, Arnold GW. Surface nitridation of phosphate glass. J. Non-Crystalline Solids 1990; 120: 172-177.

9. Fletcher JP, Risbud, SH, Kirkpatrick RJ. MAS-NMR structural analysis of barium aluminofluoriphosphate glasses with and without nitridation. J. Materials Research 1990; 5-04: 835-840.

10. Niida H, Takahashi M, Uchino T, Yoko T. Preparation and structure of organicinorganic hybrid precursors for new type low-melting glasses. J. Non-Crystalline Solids 2002; 306: 292-299.

11. Urman K, Otaigbe JU. New phosphate glass/polymer hybrids-Current status and future prospects. Progress in Polymer Science 2007; 32: 1462-1498.

12. Urman K, Otaigbe JU. Novel phosphate glass/polyamide 6 hybrids: miscibility, crystallization kinetics, and mechanical properties. J. Polymer Science Part B 44 (2006) 441-450.

13. Urman K, Madbouly S, Otaigbe JU. Unusual accelerated molecular relaxations of a tin fluorophosphate glass/polyamide 6 hybrid studied by broadband dielectric spectroscopy. Polymer 48 (2006) 1659-66. 
14. Urman $\mathrm{K}$ and Otaigbe JU. Rheology of tin fluorophosphate glass/polyamide 12 hybrids in the low concentration regime. Rheology 51 (2007) 1171.

15. Urman K, Iverson D, Otaigbe JU. A study of the effects of processing conditions on the structure and properties of phosphate glass/polyamide 12 hybrid materials. J. Applied Polymer Science 105 (2006) 1297-1308.

16. Urman K, Schweizer T, Otaigbe JU. Uniaxial elongation flow effects and morphology development in LDPE/phosphate glass hybrids. Rheologica Acta 46 (2007) 989-1001.

17. Berghaus D G, Peacock H B, Deformation and strain analysis for high extrusion ratios and elevated temperatures, Experimental Mechanics, 25 (1985) 301-307)

18. Van Puyvelde P, Mouldenaers P, Rheology and morphology development in immiscible polymer blends, Rheology Reviews 2005, pp 101-145.

19. Elemans P H M, Bos, H L, Janssen, J M H, Meijer, H E H, Transient phenomena in dispersive mixing, Chem. Eng., 48 (1993) 267-276.

20. Socrates G., Infrared and Raman Characteristic group Frequencies, Tables and Charts, $3^{\text {rd }}$ Edition, Wiley (2001).

21. Bellamy L.J., Advances in Infrared Group Frequencies, Methuen (1968) London.

22. Rubin II, editor. Handbook of plastic materials and technology. New York: Wiley; 1990.

23. Nishi T, Wang T. Melting Point Depression and Kinetic Effects of Cooling on Crystallization in Poly(vinylidene fluoride)-Poly(methyl methacrylate) Mixtures. Macromolecules 1975; 8 (6): 909-915.

24. Mackay ME, Dao TT, Tuteja A, Ho, DL, Van Horn, B, Kim, HC and Hawker, CJ, Nanoscale effects leading to non-Einstien-like decrease in viscosity, Nature Materials 2003; $2: 762-766$.

25. Nishi T, Wang T. Melting Point Depression and Kinetic Effects of Cooling on Crystallization in Poly(vinylidene fluoride)-Poly(methyl methacrylate) Mixtures. Macromolecules 1975; 8 (6): 909-915. 
26. Flory PJ, Principles of Polymer Chemistry, Cornell University Press, Ithaca, New York, 1953.

27. Strange J.R., Rahman M., Smith E.G., Characterization of porous solids by NMR, Phys. Rev. Lett.,71 (1993) 3589-3591.

Table 1. Compositions and properties of Tin-Fluoride Phosphate glasses.

\begin{tabular}{|c|c|c|c|c|c|c|}
\hline \multirow{2}{*}{$\begin{array}{l}\text { Glass } \\
\text { identity }\end{array}$} & \multicolumn{3}{|c|}{ Molar composition } & \multirow{2}{*}{$\begin{array}{l}\text { Density } \\
\left(\mathrm{kg} / \mathrm{m}^{3}\right)\end{array}$} & \multirow{2}{*}{$\begin{array}{l}\mathrm{T}_{\mathrm{g}} \\
\left({ }^{\circ} \mathrm{C}\right)\end{array}$} & \multirow{2}{*}{$\begin{array}{l}\mathrm{T}_{\mathrm{s}} \\
\left({ }^{\circ} \mathrm{C}\right)\end{array}$} \\
\hline & $\mathrm{P}_{2} \mathrm{O}_{5}$ & $\mathrm{SnO}$ & $\mathrm{SnF}_{2}$ & & & \\
\hline GlassA & 42 & 28 & 30 & $3230 \pm 220$ & $162.5 \pm 5.5$ & $350 \pm 12.00$ \\
\hline GlassB & 36 & 24 & 40 & $3500 \pm 90$ & $157.5 \pm 5.5$ & $331 \pm 14.00$ \\
\hline GlassC & 30 & 20 & 50 & $3560 \pm 40$ & $125.0 \pm 4.5$ & $310 \pm 12.00$ \\
\hline GlassD & 24 & 16 & 60 & $3920 \pm 60$ & $106.0 \pm 4.5$ & $289 \pm 12.00$ \\
\hline
\end{tabular}

Table 2: Equilibrium force Fe of PA11 and hybrid extrudates.

\begin{tabular}{l|c|c}
\hline Glass-PA11 Hybrids & $\begin{array}{c}\text { Glass content } \\
(\text { vol } \%)\end{array}$ & $\begin{array}{c}\text { Equilibrium Force, Fe } \\
(\mathrm{N})\end{array}$ \\
\hline Neat PA11 & 0 & $1932 \pm 432$ \\
GlassA/PA11 & 20 & $1882 \pm 234$ \\
GlassB/PA11 & 20 & $1759 \pm 367$ \\
GlassC/PA11 & 20 & $612 \pm 135$ \\
GlassD/PA11 & 20 & $348 \pm 72$ \\
GlassD/PA11 & 10 & $698 \pm 82$ \\
GlassD/PA11 & 5.0 & $1075 \pm 56$ \\
GlassD/PA11 & 2.5 & $1317 \pm 178$ \\
\hline
\end{tabular}

Table 3: Glass transition temperature of PA11 and hybrids with 20 vol\% glass measured by DMA.

\begin{tabular}{lllll}
\hline Blend & $\mathrm{SnF}_{2}$ in glass [vol\%] & Glass [vol\%] & $\mathrm{Tg}\left[{ }^{\circ} \mathrm{C}\right]$ & St. Dev. \\
\hline PA 11 & - & 0 & 63.5 & \pm 7.5 \\
GlassA/PA11 & 30 & 20 & 59.0 & \pm 4.5 \\
GlassB/PA11 & 40 & 20 & 57.5 & \pm 9.5 \\
GlassC/PA11 & 50 & 20 & 56.0 & \pm 6.5 \\
GlassD/PA11 & 60 & 20 & 38.5 & \pm 4.5 \\
\hline
\end{tabular}


Table 4: Decomposition temperatures $\left({ }^{\circ} \mathrm{C}\right)$ for neat PA11 and hybrids with 20 vol\% glass by TGA

\begin{tabular}{lllll}
\hline Blend & $\begin{array}{l}\text { At 5\% of } \\
\text { mass loss }\end{array}$ & $\begin{array}{l}\text { At 10\% of } \\
\text { mass loss }\end{array}$ & $\begin{array}{l}\text { Inflection } \\
\text { Point }\end{array}$ & $\begin{array}{l}\text { At 50\% of } \\
\text { mass loss }\end{array}$ \\
\hline PA11 & $415 \pm 6$ & $426 \pm 5$ & $447 \pm 5$ & $454 \pm 4$ \\
GlassA/PA11 & $411 \pm 3$ & $425 \pm 3$ & $442 \pm 5$ & $478 \pm 5$ \\
GlassB/PA11 & $423 \pm 7$ & $438 \pm 5$ & $452 \pm 4$ & $477 \pm 4$ \\
GlassC/PA11 & $422 \pm 4$ & $439 \pm 7$ & $455 \pm 6$ & $476 \pm 3$ \\
GlassD/PA11 & $426 \pm 3$ & $441 \pm 6$ & $460 \pm 5$ & $477 \pm 6$ \\
\hline
\end{tabular}

Table 5: Dissolution rates of PA11 and hybrids with 20vol\% glass. The samples were immersed in distilled water at $37^{\circ} \mathrm{C}$ and the mass loss measured with respect to time.

\begin{tabular}{lllll}
\hline Blend & $\begin{array}{l}\text { Dissolution Rate } \\
\left(\mathrm{mg} \mathrm{cm}^{-2} \mathrm{~h}^{-1}\right)\end{array}$ & $\begin{array}{l}\mathrm{SnF}_{2} \text { in the } \\
\text { glass }(\mathrm{mol} \%)\end{array}$ & $\begin{array}{l}\mathrm{Tg} \text { of glass } \\
\left({ }^{\circ} \mathrm{C}\right)\end{array}$ & $\begin{array}{l}\text { Ts of glass } \\
\left({ }^{\circ} \mathrm{C}\right)\end{array}$ \\
\hline PA11 & $0.0061 \pm 0.0005$ & 0 & - & - \\
GlassA/PA11 & $0.0058 \pm 0.001$ & 30 & 162 & 350 \\
GlassB/PA11 & $0.0049 \pm 0.0015$ & 40 & 157 & 331 \\
GlassC/PA11 & $0.0044 \pm 0.0008$ & 50 & 125 & 310 \\
GlassD/PA11 & $0.0037 \pm 0.0007$ & 60 & 106 & 289 \\
\hline
\end{tabular}

Table 6: Equilibrium melting points of PA11 and hybrids determined by Hoffman-Weeks approach.

\begin{tabular}{lllll}
\hline Blend & Glass name & Glass [vol\%] & $\operatorname{Tm}^{0}\left[{ }^{\circ} \mathrm{C}\right]$ & St. Dev. \\
\hline PA 11 & - & 0 & 200.5 & \pm 2.5 \\
20GlassA/PA11 & GlassA & 20.0 & 187.5 & \pm 1.6 \\
20GlassB/PA11 & GlassB & 20.0 & 193.0 & \pm 1.3 \\
20GlassC/PA11 & GlassC & 20.0 & 188.8 & \pm 1.2 \\
2.5GlassD/PA11 & GlassD & 2.5 & 189.2 & \pm 1.3 \\
5GlassD/PA11 & GlassD & 5.0 & 189.0 & \pm 0.6 \\
10GlassD/PA11 & GlassD & 10.0 & 188.3 & \pm 0.2 \\
20GlassD/PA11 & GlassD & 20.0 & 186.5 & \pm 0.7 \\
\hline
\end{tabular}


Table 7: Mechanical properties for neat PA11 and polyamide11 - phosphate glass hybrids.

\begin{tabular}{lcccclc}
\hline \multicolumn{1}{c}{ Blend } & $\begin{array}{c}\text { Yield } \\
\text { Strength } \\
(\mathrm{MPa})\end{array}$ & $\begin{array}{c}\text { Tensile } \\
\text { Strength } \\
(\mathrm{MPa})\end{array}$ & $\begin{array}{c}\text { Flexural } \\
\text { Strength } \\
(\mathrm{MPa})\end{array}$ & $\begin{array}{c}\text { Elongation Young's } \\
\text { at break } \\
(\%)\end{array}$ & $\begin{array}{c}\text { Modulus } \\
(\mathrm{MPa})\end{array}$ & $\begin{array}{c}\text { Flexural } \\
\text { Modulus } \\
(\mathrm{MPa})\end{array}$ \\
\hline PA 11 & $20.4 \pm 0.5$ & $32.9 \pm 0.2$ & $34.5 \pm 5.3$ & $310 \pm 3.7$ & $1008 \pm 54$ & $828 \pm 35$ \\
GlassA/PA11 & $20.0 \pm 0.4$ & $22.0 \pm 0.6$ & $26.0 \pm 3.6$ & $2.2 \pm 0.7$ & $1265 \pm 78$ & $947 \pm 58$ \\
GlassB/PA11 & $14.9 \pm 0.1$ & $29.5 \pm 0.7$ & $32.5 \pm 2.2$ & $11.9 \pm 2.6$ & $746 \pm 14$ & $954 \pm 59$ \\
GlassC/PA11 & $25.6 \pm 0.2$ & $33.7 \pm 0.3$ & $36.1 \pm 3.7$ & $90.8 \pm 33$ & $1172 \pm 56$ & $970 \pm 45$ \\
GlassD/PA11 & $26.3 \pm 1.0$ & $34.9 \pm 0.4$ & $37.4 \pm 3.4$ & $17.9 \pm 7.6$ & $1508 \pm 28$ & $1213 \pm 88$ \\
\hline
\end{tabular}




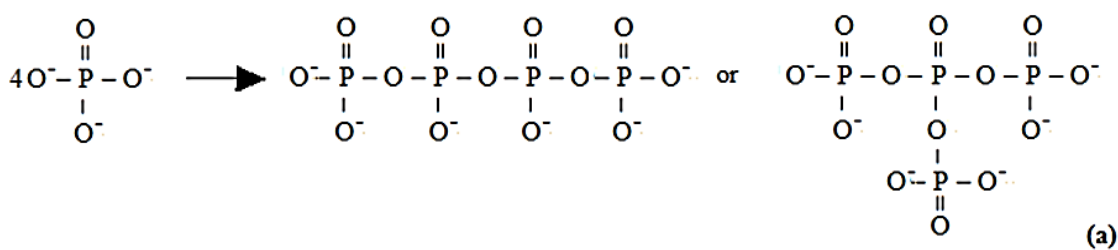

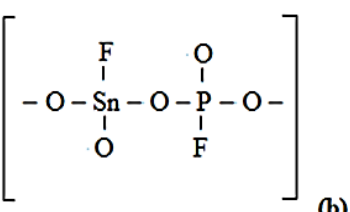

$$
\left[\stackrel{\mathrm{O}}{\mathrm{NH}-\mathrm{C}}-\left(\mathrm{CH}_{2}\right)_{10}-\right]_{\mathrm{n}}
$$

Fig. 1. Structure of phosphate glasses: (a) tetrahedral phosphate anion $\mathrm{PO}^{3-}$ and polymerization of phosphates giving polyphosphate anions; (b) repeat unit of tin fluoride phosphate glass; (c) repeat unit of PA11.

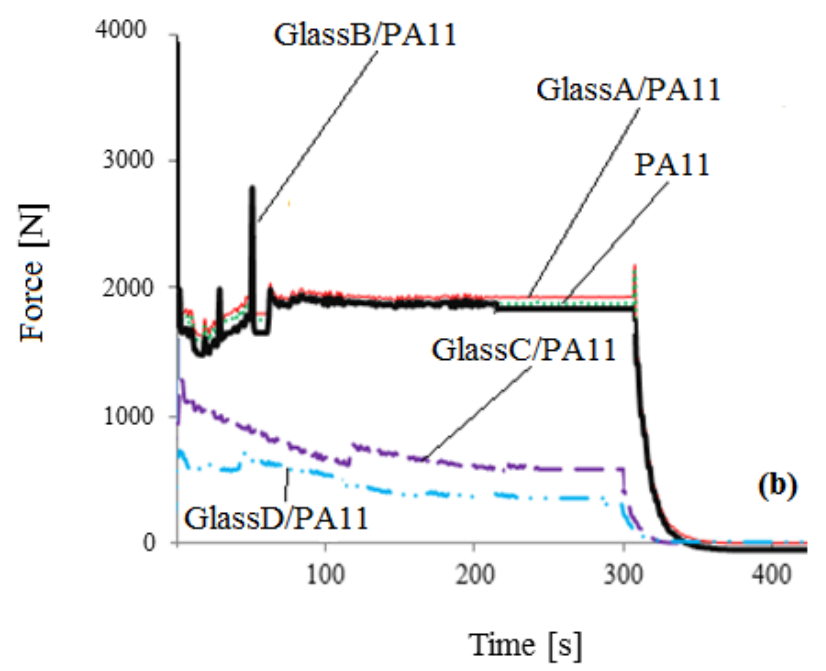

Fig. 2. Force-time profiles during extrusion of PA11 and hybrids containing constant 20 vol\% of Glass A, GlassB, GlassC and GlassD.
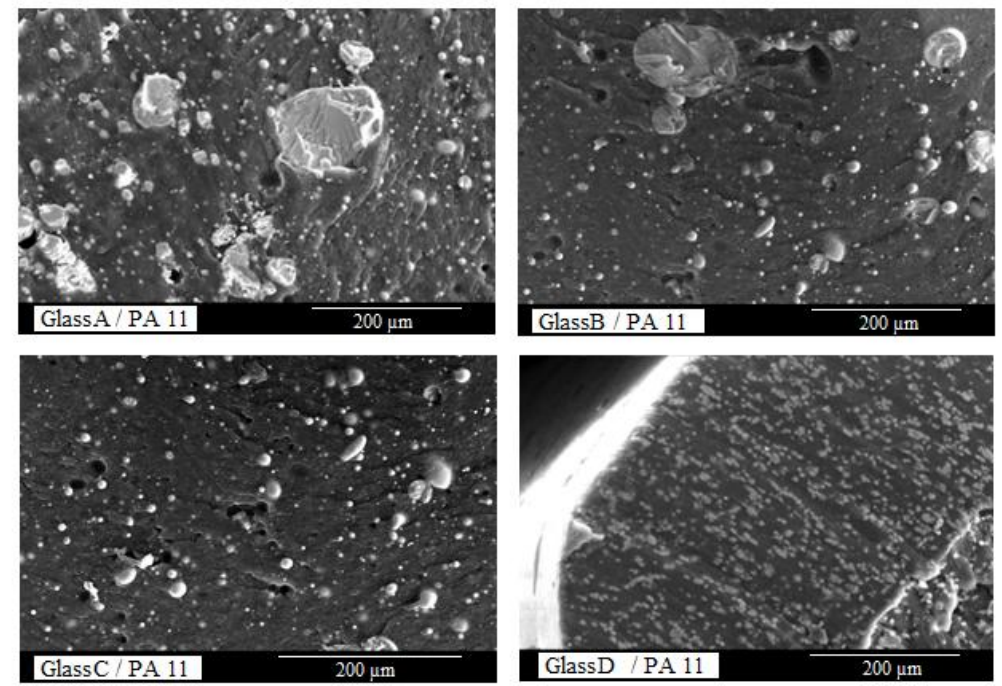

Fig. 3. Electron micrographs of cross sections of GlassA/PA11, Glass B/PA11, GlassC/PA11 and GlassD/PA11 containing 20 vol\% of glass 


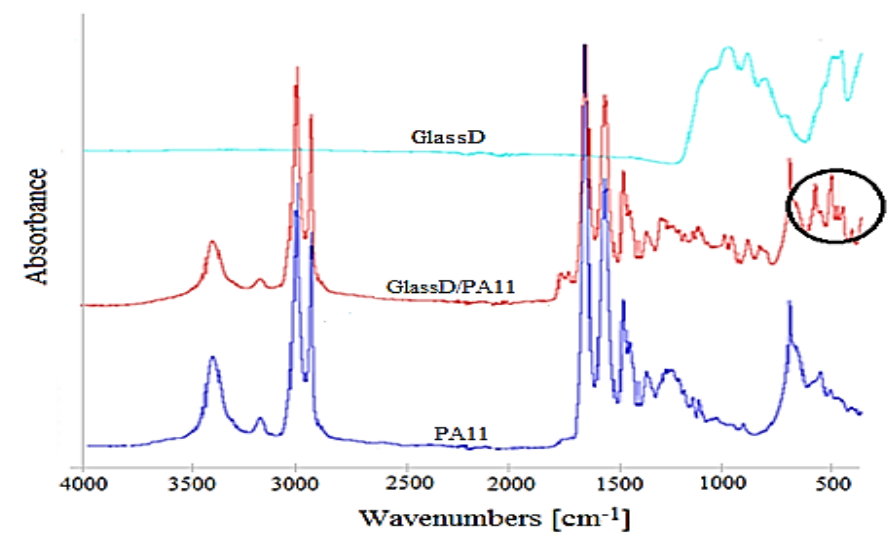

Fig. 4. FTIR spectra of PA11, GlassD and GlassD/PA11 hybrid with addition of 20 vol\% of glass.

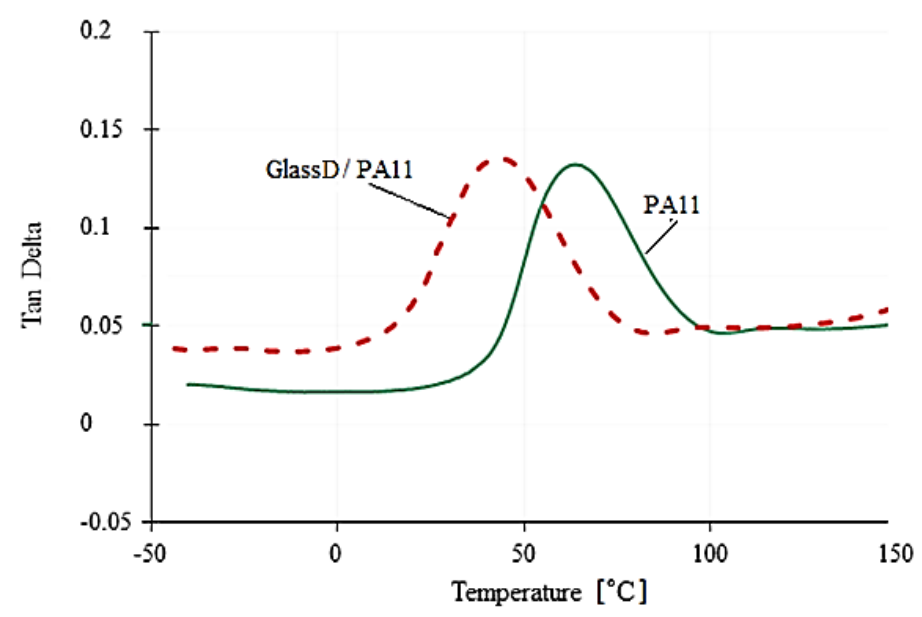

Fig. 5. Dissipation factor tan $\delta$ versus temperature from torsional DMA for pure PA11 and GlassD/PA11 hybrid filled with 20 vol\% of GlassD

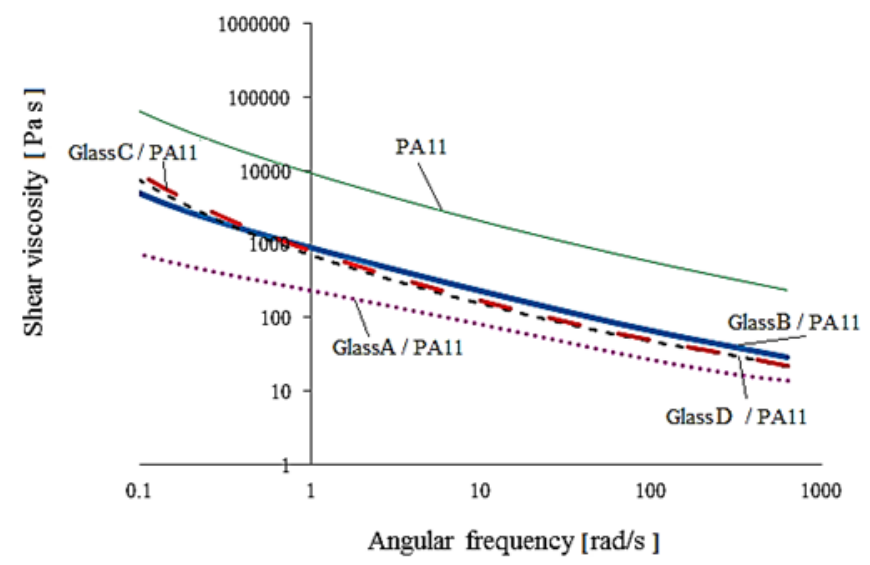

Fig. 6. Complex shear viscosity versus angular frequency of PA11, GlassA/PA11, GlassB/PA11 


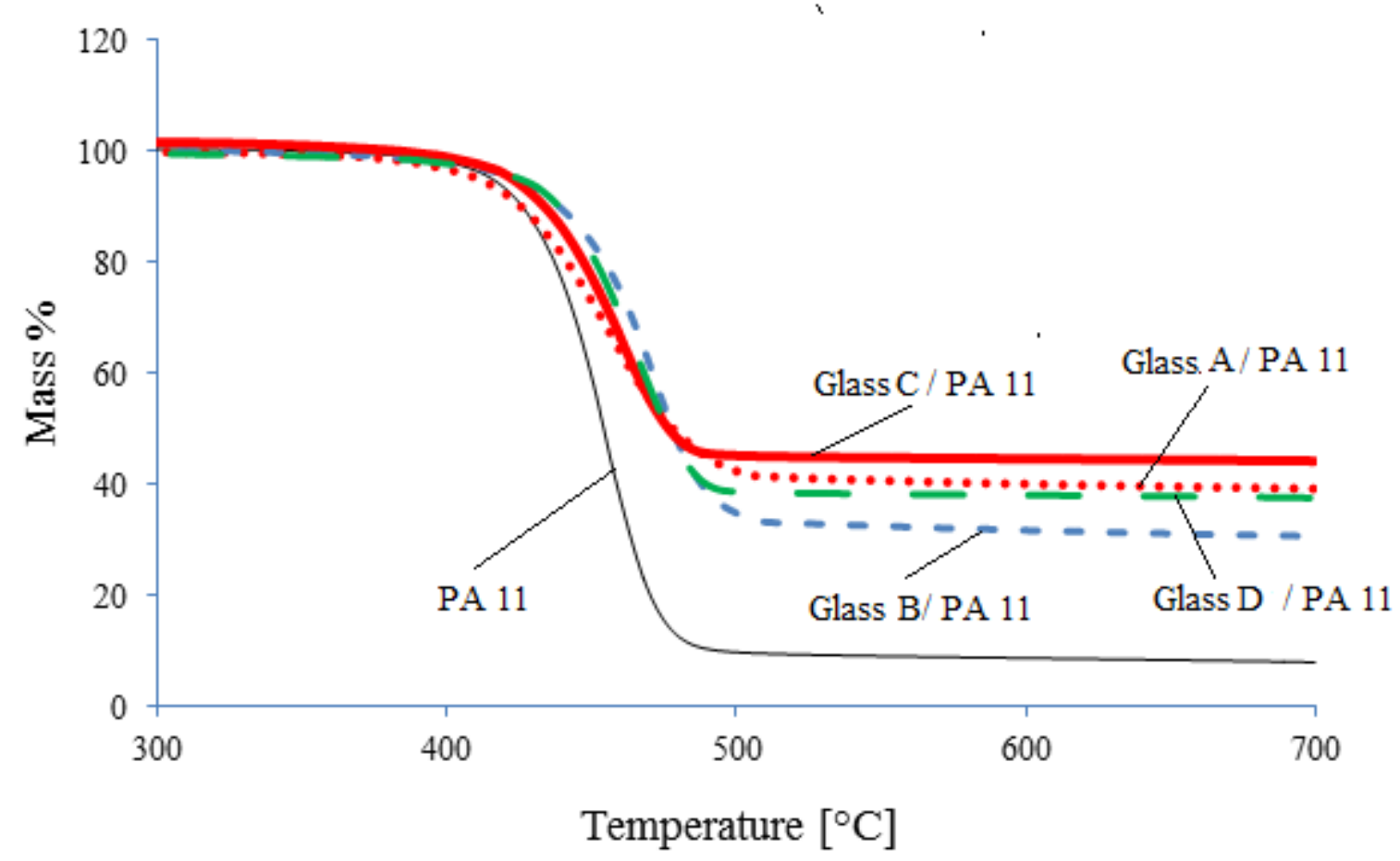

Fig 7. Thermogravimetric analysis data of PA 11 and hybrids with 20 vol\% glass content measured at a scan rate of $10^{\circ} \mathrm{C} / \mathrm{min}$ in air

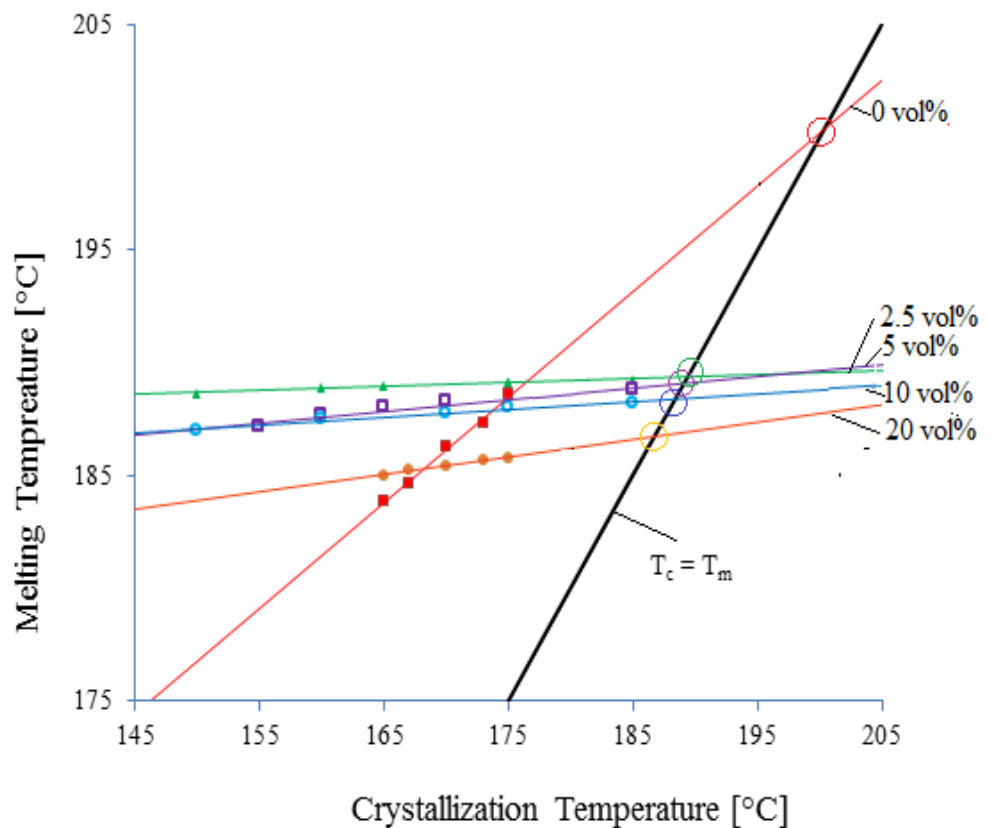

Fig 8: Melting point versus crystallization temperature of GlassD/PA11 hybrids with glass content in the range 0-20 vol\% according to the Hoffman-Weeks approach. 


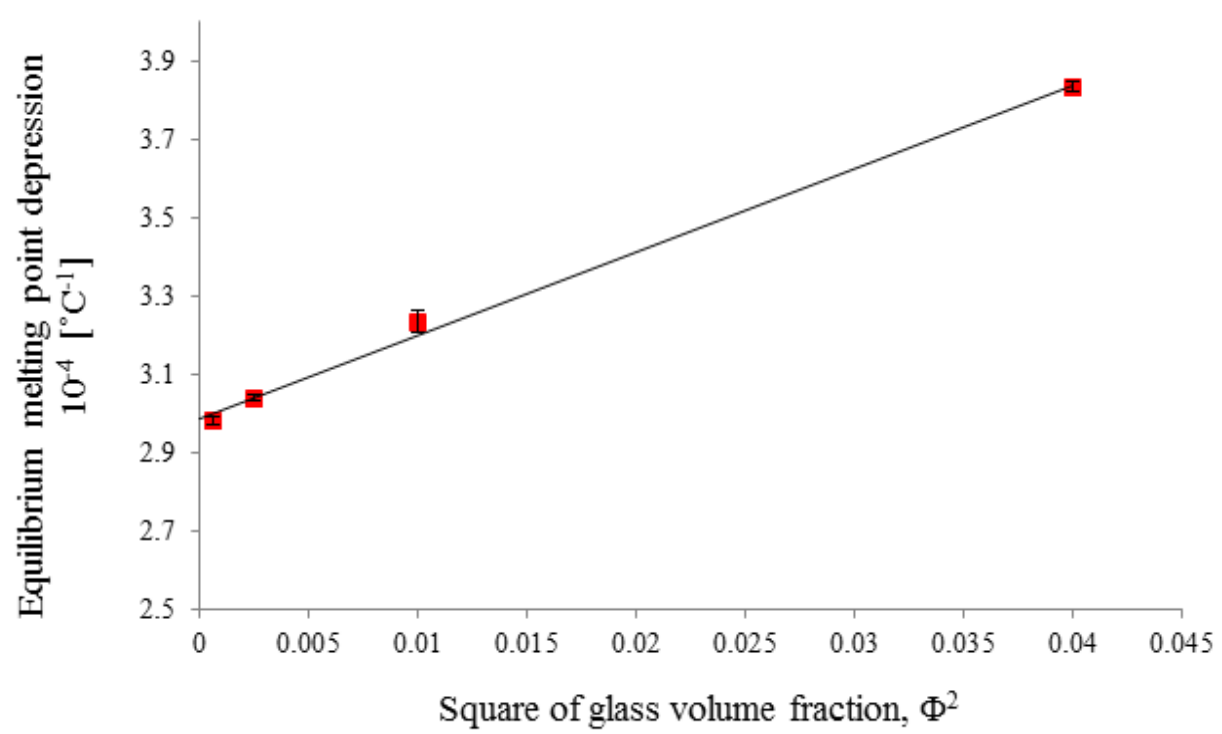

Fig.9. Melting-point depression of hybrids as a function of the square of Glass D volume fraction. 
